\title{
Universiteit
}

Leiden

The Netherlands

\section{GENETIC RISK FACTORS FOR TYPE 2 DIABETES MELLITUS AND RESPONSE TO SULFONYLUREA TREATMENT}

Swen, J.J.; Wessels, J.A.; Assendelft, W.J.; Guchelaar, H.J.

\section{Citation}

Swen, J. J., Wessels, J. A., Assendelft, W. J., \& Guchelaar, H. J. (2011). GENETIC RISK FACTORS FOR TYPE 2 DIABETES MELLITUS AND RESPONSE TO SULFONYLUREA TREATMENT. Clinical Pharmacology And Therapeutics, 89, S76S76. Retrieved from https://hdl.handle.net/1887/117650

Version:

Not Applicable (or Unknown)

License: $\quad$ Leiden University Non-exclusive license

Downloaded from: $\quad$ https://hdl.handle.net/1887/117650

Note: To cite this publication please use the final published version (if applicable). 


\title{
Genetic risk factors for type 2 diabetes mellitus and response to sulfonylurea treatment
}

\author{
Jesse J. Swen ${ }^{\mathrm{a}}$, Henk-Jan Guchelaar ${ }^{\mathrm{a}}$, Renée F. Baak-Pablo ${ }^{\mathrm{a}}$, \\ Willem J.J. Assendelft ${ }^{\mathrm{b}}$ and Judith A.M. Wessels ${ }^{\mathrm{a}}$
}

\begin{abstract}
Objective After the identification of type 2 diabetes mellitus (T2DM) risk alleles from genome-wide association studies, models have been developed to identify subjects at high risk to develop T2DM. We hypothesize that a panel of 20 repeatedly associated T2DM risk alleles influences response to sulfonylureas (SUs).
\end{abstract}

\section{Methods Two hundred and seven incident SU} (tolbutamide, glibenclamide, glimepiride, gliclazide) users with T2DM were recruited from four primary care centers. A genetic risk score per patient was calculated based on the number of risk-alleles. With this score, patients were categorized into three predefined genetic risk groups. The effect of the genetic risk group on the achievement of stable SU dose, prescribed stable SU dose, and time to stable SU dose was analyzed.

Results Carriers of more than 17 T2DM risk alleles had a 1.7-fold reduced likelihood to achieve stable SU dose $(P=0.044)$. No significant effect of the number of T2DM risk alleles on prescribed dose was found. Carriers of more than 17 T2DM risk alleles showed a marginally significant

\section{Introduction}

The incidence of type 2 diabetes mellitus (T2DM) is increasing at an alarming rate. Worldwide, the number of patients is expected to increase from 171 million in 2000 to 366 million in 2030 [1]. The therapeutic goal of treating T2DM patients is to prevent or delay long-term microvascular and macrovascular complications by achieving the best possible glycemic control.

Sulfonylureas (SUs) are part of the mainstay of treatment with oral antidiabetic drugs. Tolbutamide, glibenclamide (glyburide), gliclazide, and glimepiride are the most commonly used representatives of this group. These drugs act by closing the pancreatic $\beta$-cell potassium channels, stimulating insulin secretion [2]. SUs are initiated at a low dose and escalated to the optimal dose with intervals of 2-4 weeks until the glycemic target $(\mathrm{HbA1c}<7 \%)$ is achieved. However, there is significant interpatient variability in response to SUs, with approximately $10-20 \%$ of the patients experiencing primary failure (decrease in fasting glucose level $<1.1 \mathrm{mmol} / \mathrm{l}$ )

\footnotetext{
Supplemental digital content is available for this article. Direct URL citations appear in the printed text and are provided in the HTML and PDF versions of this article on the journal's Website (www.pharmacogeneticsandgenomics.com).
}

increased time to stable dose (hazard ratio: $0.81 ; 95 \%$ confidence interval, 0.75-1.01, $P=0.058$ ).

Conclusion T2DM risk alleles are associated with response to SUs in primary care T2DM patients. This suggests that individualization of T2DM treatment according to genetic profile may be an opportunity to improve clinical outcome. Pharmacogenetics and Genomics 21:461-468 (c) 2011 Wolters Kluwer Health | Lippincott Williams \& Wilkins.

Pharmacogenetics and Genomics 2011, 21:461-468

Keywords: glibenclamide, gliclazide, glimepiride, pharmacogenetics, sulfonylureas, tolbutamide, type 2 diabetes mellitus

Departments of ${ }^{a}$ Clinical Pharmacy and Toxicology and ${ }^{b}$ Public Health and Primary Care, Leiden University Medical Center, Leiden, The Netherlands

Correspondence to Dr Henk-Jan Guchelaar, PharmD, PhD, Department Clinical Pharmacy and Toxicology, Leiden University Medical Center, Albinusdreef 2, PO Box 9600, NL 2300 RC Leiden, The Netherlands Tel: + 3171526 2790; fax: + 31715248101 ; e-mail: h.j.guchelaar@lumc.nl

Received 19 November 2010 Accepted 6 April 2011

and a similar percentage having an above average response (mean reduction HbA1c 1.5-2\%) [3-5].

With the completion of multiple genome-wide association studies (GWAS) the knowledge of the complex genetic background of T2DM has increased. These studies report associations between genetic variants and the risk for the development of T2DM. A panel of 20 T2DM associated single nucleotide polymorphisms (SNPs) comprising 19 genes out of the GWAS data appears, that has been replicated in several studies [6-17]. These SNPs are used in models with the ultimate goal to identify subjects at high risk to develop T2DM. Although marginally, the addition of genetic information to clinical T2DM risk factors increased the ability to predict future diabetes [18-24].

From the panel of 20 T2DM risk-associated SNPs, the majority is involved in the process of insulin release from the pancreatic $\beta$-cells (Table 1 ). As SUs act by stimulating insulin secretion, response to $\mathrm{SU}$ treatment may also be influenced by these genetic variants. Indeed, two of the 19 T2DM risk-associated genes, encoding KCNJ11 and TCF7L2, have been previously correlated with variation in SU response [4]. Furthermore, in subjects 
Table 1 Selected single nucleotide polymorphisms associated with type 2 diabetes mellitus

\begin{tabular}{|c|c|c|c|c|c|c|}
\hline Gene & rs number & Chromosome & Risk allele & Year & Mechanism & References \\
\hline $\mathrm{NOTCH} 2$ & rs10923931 & 1 & $\mathrm{~T}$ & 2008 & Unknown & {$[9,19-24]$} \\
\hline THADA & rs7578597 & 2 & $\mathrm{~T}$ & 2008 & Unknown & {$[9,19-24]$} \\
\hline IGF2BP2 & rs4402960 & 3 & $\mathrm{~T}$ & 2007 & $\beta$-cell dysfunction & {$[8,9,11-13,18-24]$} \\
\hline PPARG & rs1801282 & 3 & $\mathrm{C}$ & 2000 & Insulin sensitivity & {$[8,9,11-13,19-24]$} \\
\hline ADAMTS9 & rs4607103 & 3 & $\mathrm{C}$ & 2008 & Unknown & {$[9,19-24]$} \\
\hline WFS1 & rs10010131 & 4 & $G$ & 2007 & Unknown & {$[9,13,18-20,22,24]$} \\
\hline$C D K A L 1$ & rs7754840 & 6 & $\mathrm{C}$ & 2007 & $\beta$-cell dysfunction & {$[7-9,11-13,18-24]$} \\
\hline$J A Z F 1$ & rs864745 & 7 & A & 2008 & $\beta$-cell dysfunction & {$[9,19-24]$} \\
\hline SLCЗОA8 & rs13266634 & 8 & $\mathrm{C}$ & 2007 & $\beta$-cell dysfunction & {$[7-9,11-14,18-24]$} \\
\hline \multirow[t]{2}{*}{$C D K N 2 A / C D K N 2 B$} & rs10811661 & 9 & $\mathrm{~T}$ & 2007 & $\beta$-cell dysfunction & {$[8,9,11-13,18-24]$} \\
\hline & rs564398 & 9 & $A$ & & & {$[8,18,20,24]$} \\
\hline TCF7L2 & rs7903146 & 10 & $\mathrm{~T}$ & 2006 & $\beta$-cell dysfunction & {$[7-9,11-14,18-24]$} \\
\hline HHEXIIDE & rs1111875 & 10 & $\mathrm{G}$ & 2007 & $\beta$-cell dysfunction & {$[7-9,11-14,18-24]$} \\
\hline$C D C 123 / C A M K 1 D$ & rs12779790 & 10 & $\mathrm{G}$ & 2008 & Unknown & {$[9,19-24]$} \\
\hline KCNJ11 & rs5219 & 11 & $\mathrm{~T}$ & 2003 & $\beta$-cell dysfunction & {$[8,9,11-13,19-24]$} \\
\hline KCNQ1 & rs2237892 & 11 & $\mathrm{C}$ & 2008 & $\beta$-cell dysfunction & {$[10,17,24]$} \\
\hline MTNR1B & rs10830963 & 11 & $\mathrm{G}$ & 2009 & Disturbance of circadian rhythm & {$[6,16,24]$} \\
\hline TSPAN8/LGR5 & rs7961581 & 12 & $\mathrm{C}$ & 2008 & Unknown & {$[9,19-24]$} \\
\hline FTO & rs8050136 & 16 & A & 2007 & Obesity & {$[8,9,11,13,19-22,24]$} \\
\hline$H N F-1 \beta$ (TCF2) & rs757210 & 17 & $A$ & 2007 & $\beta$-cell dysfunction & {$[15,20,21,24]$} \\
\hline
\end{tabular}

analyzed for genetic variation in the genes TCF7L2, PPARG, FTO, KCNJ11, NOTCH2, WFS1, CDKAL1, IGF2BP2, SLC30A8, JAZF1, HHEX, it was reported that subjects with 12 or more T2DM risk alleles did not increase their insulin secretion to compensate for the increased insulin resistance as efficiently as those with 8 or less risk alleles [19]. Therefore, patients with a greater number of risk alleles may show less response to $\mathrm{SU}$ treatment and individualization of T2DM treatment according to genetic profile may be an opportunity to improve clinical outcome.

We hypothesize that the genetic variants associated with the development of T2DM are also associated with response to SU treatment. Therefore, we investigated the effect of T2DM risk alleles on the response to $\mathrm{SU}$ treatment in T2DM patients in a primary care setting.

\section{Methods}

\section{Study setting}

In the Netherlands the general practitioner (GP) plays a central role in the provision of health care. Patients are listed with one GP who is consulted for all healthcare problems and indicates whether a referral to secondary care is appropriate. Typically, the GP keeps an electronic patient record (EPR) that covers all medical information concerning the patient including prescription information and reports from laboratories and specialists. GP's have adopted the practice guideline T2DM of the Dutch College of General Practitioners [25]. Tailoring the treatment to the individual patient is an important part of the therapy.

\section{Cohort ascertainment}

A total of 207 T2DM patients from four universityaffiliated primary care centers (17 GPs) located in the vicinity of Leiden, the Netherlands were recruited. The ascertainment of the cohort has been described in detail previously [26]. In brief, patients that had at least one prescription of tolbutamide, glibenclamide, glimepiride, or gliclazide between January 1992 and June 2008, were at least 18 years of age and without insulin use at the time of first SU prescription, and had at least 270 days of follow-up registered in the EPR, were included. Ethnicity was not routinely recorded in the EPR but most patients in the Netherlands are from European ancestry. Patients received a written invitation by mail from their GP. Of the 472 invited patients, $222(47 \%)$ agreed to participate (see Fig. S1, Supplemental digital content 1, http://links.lww. com/FPC/A263, cohort ascertainment). After consent, a saliva collection kit (DNA Genotek Inc., Ottawa, Ontario, Canada) was mailed. The study was approved by the ethics committee of the Leiden University Medical Center.

\section{Genotyping}

We selected a panel of 20 SNPs in 19 genes that have been associated with the development of T2DM in at least three GWAS and were consistently replicated in later studies aimed at estimating the predictive value of these SNPs on the development of T2DM [6-24]. The selected SNPs are listed in Table 1. DNA was isolated from the saliva according to the protocol provided by the manufacturer (DNA Genotek Inc.). Taqman genotyping assays for 19 SNPs were designed by and obtained from Applied Biosystems (Applied Biosystems, Nieuwerkerk aan den IJssel, the Netherlands). SNP rs757210 could not be designed as a Taqman genotyping assay and therefore was genotyped by pyrosequencing (Isogen Life Science, Maarssen, the Netherlands). Taqman genotyping assays were performed on the LightCycler 480 II Real-Time PCR System (Roche Diagnostics, Almere, the Netherlands) according to standard procedures. Genotyping was performed without knowledge of the clinical data. We obtained an average genotyping success rate of more than $95 \%$. As a quality control $5 \%$ of the samples were 
genotyped in duplicate for all assays and no inconsistencies were observed. Five patients were excluded for quality reasons (genotype call rate $\leq 80 \%$ ). All SNPs were in Hardy-Weinberg equilibrium $(P>0.05)$, with the exception of rs2237892 $(P=0.011)$. This is most probably ascribed to the very low minor allele frequency of rs2237892, which was 0.025 in our study and comparable with previously reported minor allele frequencies of 0.056-0.075 (http://wwwencbi.nlm.nih.goo/projects/SNP/snp_ ref.cgi? $r s=2237892)$ accessed 5 October 2010 .

\section{Definition of effect}

For each patient a cumulative genetic risk score was calculated based on the number of present risk alleles. Each person could have 0,1 , or 2 of them for each SNP, resulting in a theoretical individual cumulative risk score between 0 and 40. This approach assumes an equal and additive effect of each allele on the risk of T2DM. To allow categorization of patients, we predefined three genetic risk groups on the basis of the frequency distribution of risk alleles. We defined a low genetic risk group and a high genetic risk group as the quintiles with the lowest and highest number of T2DM risk alleles, respectively. All other patients (three quintiles) were categorized in the intermediate risk group.

The primary endpoint of our study is the effect of the genetic risk group on achieving stable SU dose. Stable SU dose was defined as the first period of more than or equal to 270 consecutive days without $\mathrm{SU}$ dose adjustment, or initiation or adjustment of therapy with other SUs, insulin or metformin. If therapy with insulin was initiated patients were censored. The period of more than or equal to 270 days was chosen because prescriptions in the Netherlands are limited to a maximum of 90 days and more than or equal to 270 days equals three consecutive prescriptions. Stable SU dose was calculated as normalized dose by dividing the prescribed daily dose with the standard daily dose used by the Pharmaceutical Aid Committee of the Dutch Health Care Insurance Board (10 mg glibenclamide; $1000 \mathrm{mg}$ tolbutamide; $160 \mathrm{mg}$ gliclazide; $2 \mathrm{mg}$ glimepiride). Secondary endpoints of our study are the stable SU dose and the time required for dose escalation (time to stable SU dose).

\section{Statistical analysis}

The data were analyzed using the SPSS statistical package (version 16.0, SPSS, Chicago, Illinois, USA). Deviation from Hardy-Weinberg equilibrium was tested by the $\chi^{2}$ test. Achievement of stable SU dose was analyzed with the $\chi^{2}$ test and multivariate logistic regression analysis. Differences in mean stable SU dose between genetic risk groups were analyzed using the Kruskal-Wallis test and multivariate linear regression analysis. Associations between the genetic risk groups and time to stable SU dose were evaluated using the Cox survival regression analysis. Before multivariate analysis, all demographic and clinical variables were tested univariately against the selected outcome. Variables with a $P$ value of 0.1 or less, age, and sex were selected for multivariate analysis. All multivariate analyses were corrected for age and sex.

\section{Results}

Data from 202 T2DM patients were available. The range of the calculated genetic risk score was 10-26. The quintiles with the lowest $(\leq 17)$ and highest $(\geq 21)$ number of T2DM risk alleles consisted of 59 patients and 62 patients, respectively (Fig. 1). Table 2 presents the characteristics of the 202 patients. There were no differences between the different genetic risk groups observed in any of the patient characteristics except for age. Patients in the high-risk group were younger at the time of first SU prescription compared with patients in the low-risk and intermediate-risk group, respectively $(P=0.001)$. Mean follow-up was 5.9 years, reflecting that most patients $(75.2 \%)$ were included after 2000. Our patients received an average of $26 \mathrm{SU}$ prescriptions during the follow-up period with a median duration of 90 days per prescription.

The results of achieving stable SU dose and the T2DM genetic risk groups are presented in Fig. 2. Of the patients, 148 (73.3\%) achieved stable SU dose. The percentage of

Fig. 1

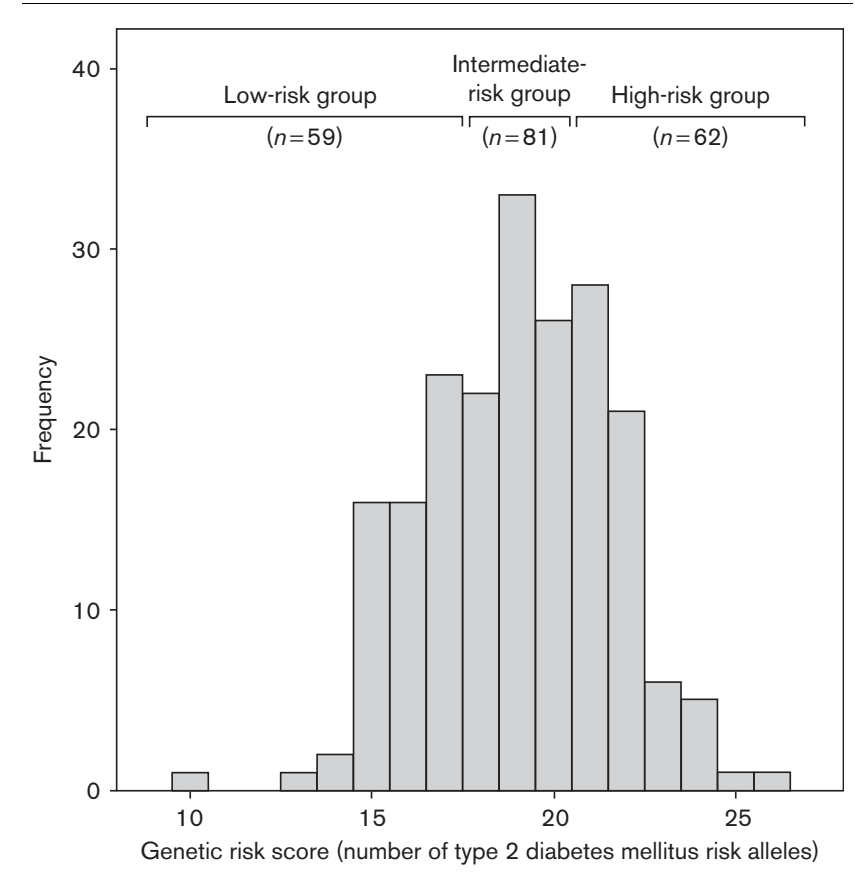

Distribution of type 2 diabetes mellitus risk alleles and subsequent classification in risk groups. Patients were categorized in three genetic risk groups. Low-risk group; quintile with the lowest $(\leq 17)$ number of T2DM risk alleles. High-risk group; quintile with the highest $(\geq 21)$ number of T2DM risk alleles. Remaining patients were categorized in the intermediate-risk group (18-20 T2DM risk alleles). 
Table 2 Characteristics of the 202 patients with type 2 diabetes mellitus in primary care

\begin{tabular}{|c|c|c|c|c|c|}
\hline \multirow[b]{2}{*}{ Variable no. $(\%)^{a}$} & \multirow[b]{2}{*}{ All patients } & \multicolumn{3}{|c|}{ Genetic risk group } & \multirow[b]{2}{*}{$P$ value } \\
\hline & & Low-risk & Intermediate-risk & High-risk & \\
\hline Subjects & 202 & $59(29.2)$ & $81(40.1)$ & $62(30.7)$ & NA \\
\hline Men & $106(52.5)$ & $30(50.8)$ & $45(55.6)$ & $31(50.0)$ & 0.77 \\
\hline Women & $96(47.5)$ & $29(49.2)$ & $36(44.4)$ & $31(50.0)$ & \\
\hline Age in years, mean (SD) & $61.4(10.7)$ & $64.0(9.5)$ & $62.6(10.4)$ & $57.3(11.1)$ & 0.001 \\
\hline Follow-up in years, mean (SD) & $5.9(3.0)$ & $6.0(3.0)$ & $5.7(3.0)$ & $6.2(3.0)$ & 0.52 \\
\hline Visits in year one (SD) & $9.6(4.7)$ & $8.6(3.7)$ & $10.0(5.2)$ & $10.0(4.6)$ & 0.35 \\
\hline Metformin use & $62(30.7)$ & $18(30.5)$ & 27 (33.3) & $17(27.4)$ & 0.75 \\
\hline Primary sulfonylurea & & & & & $0.098^{b}$ \\
\hline Glibenclamide & $12(5.9)$ & $7(11.9)$ & $1(1.2)$ & $4(6.5)$ & \\
\hline Tolbutamide & $85(42.1)$ & $18(30.5)$ & $41(50.6)$ & $26(41.9)$ & \\
\hline Gliclazide & $24(11.9)$ & 7 (11.9) & $10(12.3)$ & 7 (11.3) & \\
\hline Glimepiride & $81(40.1)$ & $27(45.8)$ & $29(35.8)$ & $25(40.3)$ & \\
\hline
\end{tabular}

Low-risk group; patients with 17 or less risk alleles. Intermediate-risk group: patients with 18-20 risk alleles. High-risk group; patients with at least 21 risk alleles. NA, not applicable; SD, standard deviation.

aunless stated otherwise.

${ }^{\mathrm{b}} \chi^{2}$ for primary sulfonylurea vs. genetic risk group.

$P$ value $<0.05$ is regarded as significant and indicated with bold font.

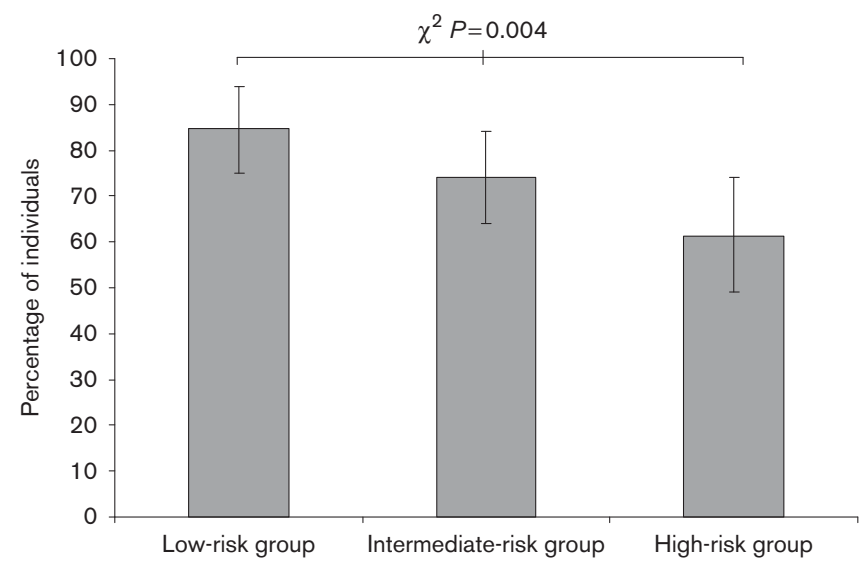

Percentage of type 2 diabetes mellitus patients that reached stable sulfonylurea dose. Low-risk group; patients with 17 or less risk alleles. Intermediate-risk group: patients with 18-20 risk alleles. High-risk group; patients with at least 21 risk alleles.

patients achieving stable SU dose was lower in the high-risk group compared with the intermediate-risk and low-risk groups (61.3 vs. 74.1 vs. $84.7 \%$, respectively, $P=0.004)$. In the multivariate logistic regression analysis age at first SU prescription, the concomitant use of metformin, and the T2DM genetic risk group were independently significantly associated with achieving stable SU dose (Table 3). The regression model explained $28.7 \%$ of the variation in achievement of stable dose. Data show that patients with a higher T2DM risk had a 1.7 -fold reduced likelihood to achieve stable $\mathrm{SU}$ dose $(P=0.044)$.

Next, the mean SU starting dose was analyzed. The mean SU starting dose for all patients was 0.61 (95\% CI: 0.58$0.65)$. As expected, no differences in SU starting dose were found between the different genetic risk groups. No differences in mean stable SU dose were found between the different T2DM genetic risk groups [low-risk group $0.90,95 \%$ confidence interval (CI) $0.75-1.05$ vs. intermediate-risk group $0.84,95 \%$ CI: $0.74-0.94$ vs. high-risk group $0.95,95 \%$ CI: $0.72-1.17, P=0.97]$. In multivariate linear regression, only the effect of the SU starting dose and sex were independently significant associated with stable SU dose, whereas the genetic risk group for T2DM was not associated with stable $\mathrm{SU}$ dose.

As SUs are escalated to the optimal dose, the effect of the genetic risk group on time to stable SU dose was evaluated. Carriers of the high-risk genetic profile ( $\geq 21$ risk alleles) had a two-fold and five-fold longer time to stable dose compared with patients with the intermediate risk (18-20 risk alleles) and low-risk profile ( $\leq 17$ risk alleles) (median time to stable SU dose $160 \mathrm{vs.}$ 59 vs. 31 days, respectively, $P=0.007$ ). In a multivariate Cox regression analysis including the factors such as age 
Table 3 Analysis of factors relevant for achieving stable sulfonylurea dose in patients with type 2 diabetes mellitus in primary care

\begin{tabular}{|c|c|c|c|c|c|c|c|}
\hline \multirow[b]{2}{*}{ Factor } & \multicolumn{4}{|c|}{ Univariate } & \multicolumn{3}{|c|}{ Multivariate $^{\mathrm{a}}$} \\
\hline & OR & $95 \% \mathrm{Cl}$ & $R^{2}$ & $P$ value & OR & $95 \% \mathrm{Cl}$ & $P$ value \\
\hline Constant & & & & & 1.81 & NA & 0.654 \\
\hline Male vs. female sex & 1.55 & $0.83-2.91$ & 0.009 & 0.17 & 1.54 & $0.72-3.29$ & 0.262 \\
\hline Effect of age (per year increase) & 1.06 & $1.03-1.09$ & 0.069 & $<0.001$ & 1.04 & $1.00-1.08$ & 0.036 \\
\hline Metformin use vs. no metformin use at stable sulfonylurea dose & 0.07 & $0.03-0.16$ & 0.238 & $<0.001$ & 0.07 & $0.03-0.17$ & $<0.001$ \\
\hline Genetic risk group (low-risk $\rightarrow$ intermediate-risk $\rightarrow$ high-risk group) & 0.54 & $0.35-0.82$ & 0.042 & 0.004 & 0.59 & $0.35-0.99$ & 0.044 \\
\hline
\end{tabular}

$\rightarrow$, stepwise increase from low-risk to intermediate-risk to high-risk group; $\mathrm{Cl}$, confidence interval; OR, odds ratio; $R^{2}$, variation in the achievement of stable dose. ${ }^{\mathrm{a}} R^{2}$ for the complete model was 0.287 .

$P$ value $<0.05$ is regarded as significant and indicated with bold font.

Fig. 3

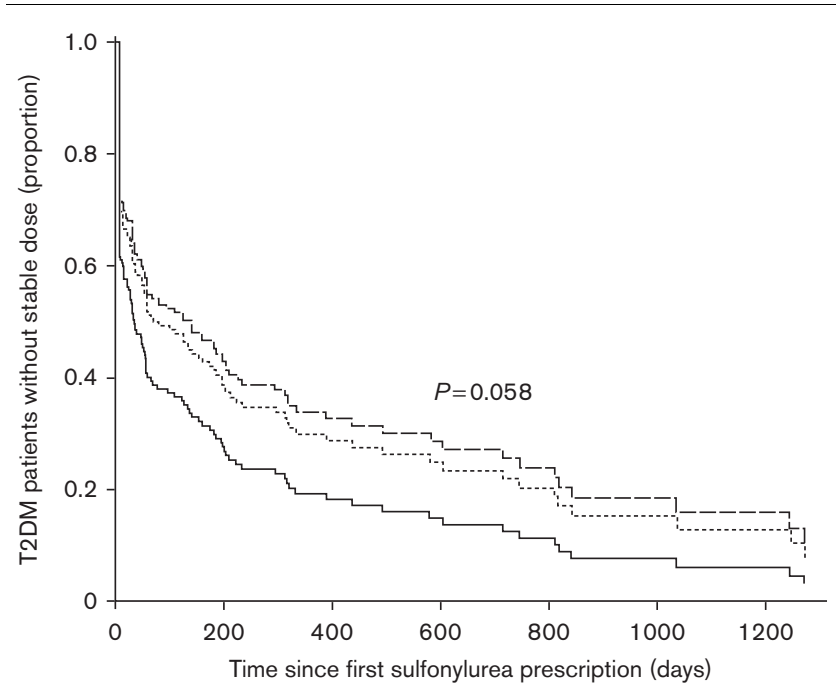

Multivariate Cox regression analysis plots of time to the first stable dose of sulfonylureas in type 2 diabetes mellitus patients in primary care.

Low-risk group (-); patients with 17 or less risk alleles. Intermediaterisk group (- - -); patients with 18-20 risk alleles. High-risk group (---); patients with at least 21 risk alleles.

on first SU prescription, sex, and the concomitant use of metformin, patients with a higher number of risk alleles showed a marginally significant increased time to stable SU dose (hazard ratio: 0.81 ; 95\% CI: 0.75-1.01, $P=0.058)$ (Fig. 3).

\section{Discussion}

To the best of our knowledge, this is the first study exploring the relationship between response to treatment with SUs and T2DM risk alleles. In this retrospective cohort study of 202 T2DM patients, patients with more than 17 risk alleles have a 1.7 -fold reduced likelihood to achieve a stable SU dose. These patients also show a marginally significant increased time to achieve stable SU dose compared with carriers of less than 17 risk alleles. However, the number of T2DM risk alleles does not seem to affect the average stable SU dose used. Therefore, our data suggest that patients with a higher number of T2DM risk alleles have a decreased and delayed response to $\mathrm{SU}$ treatment.
Drug response is determined by both pharmacokinetics and pharmacodynamics of a drug. Several groups have investigated genetic variation in genes affecting the pharmacokinetics of SU response. Two variants in CYP2C9, CYP2C9*2 and CYP2C9*3, have been associated with a decreased SU metabolism in healthy volunteers [27]. Five studies assessed the effect of these polymorphisms in T2DM patients. Presence of the CYP2C9*3 allele was associated with an increased risk for hypoglycemia $[28,29]$. Tolbutamide users with a CYP2C $9 * 2$ or CYP2C9*3 allele have been shown to have a significantly lower dose escalation compared with homozygous carriers of the CYP2C9*1 allele [30]. In a large cohort of 1073 incident SU users with T2DM Zhou et al. [31] found that carriers of the CYP2C9*2 or CYP2C9*3 allele were less likely to fail on SU monotherapy. In a recent study we found no statistically significant effect of the CYP2C9*2 or CYP2C9*3 allele on the prescribed stable dose [26].

Variation in genes associated with the pharmacodynamics of SUs in T2DM patients has received considerably less attention. Genetic variants associated with SU response have been described for some monogenic forms of diabetes [32-34]. For polygenic T2DM, variants in the genes KCNJ11, TCF7L2, ABCC8, IRS1, and NOS1AP have been associated with SU response [35-38]. Of these, only the genes KCNJ11 and TCF7L2 were reported to contribute to an increased risk for T2DM in published GWAS. KCNJ11 encodes the Kir6.2 subunit, one of the two subunits that form the ATP-sensitive potassium channel involved in insulin release. Carriership of the E23K variant of the KCNJ11 gene has been associated with failure to SU therapy, but there are some conflicting results [39-41]. Variants in the TCF7L2 gene have also been associated with SU response. In a study with 901 incident SU users, patients with the TT genotype for rs7903146 were 1.73 times less likely to be treated to lower a target HbA1c of 7\% in the first 3-12 months of treatment compared with patients with the CC genotype [42]. For a variant in linkage with rs7903146 an even larger effect (odds ratio $=1.95$ ) was reported. In this study, none of the individual risk alleles were significantly associated with the achievement of stable dose (see Table S2, Supplemental digital content 2, http:/llinks.lww.com/ FPC/A264), risk allele frequency and association with 
achievement of stable SU dose of the individual SNPs). This is most likely due to the limited sample size of our study and the probable small effect size of the individual risk alleles.

Our study has some limitations. No data were available for patients that switched to another GP or who died after 1992. Therefore, we cannot completely rule out the possibility of selection bias, although this is conceptually very unlikely. A nonresponse analysis with age, sex, type of first prescribed SU, metformin use, and GP showed no differences between participants and patients who did not consent to our study, suggesting that no selection bias has occurred on any of these parameters.

We selected stable SU dose as the primary endpoint of our analysis. Ideally macrovascular (e.g. diabetes-related death or myocardial infarction) or microvascular events (e.g. retinopathy or renal failure) would have been used. Alternatively, biomarkers related to these events, such as HbA1c or fasting plasma glucose (FPG) might have been used. However, as data concerning these parameters were not routinely recorded in the EPR, data were too sparse to be used in our analysis. Therefore, we selected stable SU dose as an alternative. Although, no SU pharmacogenetics studies have used stable SU dose as endpoint, this parameter closely reflects actual clinical practice. The time to stable SU dose analysis assumes that GPs adhere to the T2DM guideline of the Dutch College of General Practitioners and titrate SU dose in response to glucose and HbA1c levels. We have three arguments that support our assumption. Firstly, mean FPG was $7.77 \mathrm{mmol} / \mathrm{l}$ (95\% CI: $7.42-8.12, n=95$ ) for the subgroup of patients with a FPG measurement available during stable SU dose. Secondly, the adherence of GPs to guidelines is reported to be good in the Netherlands [43]. Finally, even if GPs do not adhere to the T2DM guideline, and bias would be introduced to our analysis, there is no reason to assume that the nonadherence of GPs is not divided randomly over the different genetic risk groups. Therefore, possible nonadherence does not affect the comparison of the time to stable dose between the different genetic risk groups but can only affect the absolute results of this analysis.

There are multiple known factors that predict a good response to SUs including baseline HbA1c, recently diagnosed diabetes, mild-to-moderate fasting hyperglycemia $(<12.2-13.3 \mathrm{mmol} / \mathrm{l})$, good $\beta$-cell function (high fasting C-peptide level), no history of insulin therapy, and absence of islet cell or glutamic acid decarboxylase antibodies [3]. However, for none of these factors sufficient data were available in our retrospective cohort study and we were unable to account for their effect. In addition, the available data on weight, a factor that is associated with the onset of T2DM, were too sparse to be included in the analysis as a covariate. As a consequence we cannot rule out that patients with a higher number of risk alleles also have a more severe form of T2DM that might confer to an a priori decreased probability to achieve stable SU dose. In our opinion, the only way to collect sufficient high quality data that cover all of these parameters would be to conduct a prospective observational study. Ideally such a study would include two treatment arms with pharmacological different drugs or placebo. Such a design would allow differentiating between the effect of T2DM risk alleles on disease progression and effect on treatment.

The results of different SUs were pooled in one analysis. Although SUs are generally reported to have equipotent glucose lowering effects when administered in maximally effective doses [3,5], it would be interesting to investigate if our hypothesis is valid for each of the individual SUs. However, due to the sample size of our study such a subgroup analysis was not possible.

We achieved a high success rate of genotyping with a call rate of more than $95 \%$ for all individual SNPs. After exclusion of five patients with a call rate of less than or equal to $80,0.9 \%$ of the genotype data were missing. Missing genotype data were replaced with a risk score of 0 . To test the sensitivity of our analysis for this replacement, we reanalyzed the data using two alternative approaches. First, as for some SNPs the wild-type allele is the risk allele, missing data were replaced with the score of the wild-type allele. As a result, two patients were reclassified from the low-risk to the intermediaterisk group, and one patient was reclassified from the intermediate-risk group to the high-risk group. Secondly, we excluded all patients with any missing data, resulting in the exclusion of an additional 31 (15.3\%) patients. Similar results on all end points were obtained with all approaches, except for the effect of the genetic risk score that lost statistical significance in multivariate analysis after exclusion of all patients with missing data. These sensitivity analyses indicate that our results are valid.

The analysis of the effect of the genetic risk score on $\mathrm{SU}$ response assumes that each risk allele has an equal and additive effect, both within and between loci. This is clearly a simplification of the mechanism leading to variation of SU response. However, this approach is used in all GWAS studies concerning prediction of T2DM. Until it is clear what the true effect size of individual risk alleles is, the additive genetic model is probably the most appropriate and consistent method to analyze T2DM genetic data.

We chose to compare the quintile with the lowest $(\leq 17$, $n=59$, low-risk group) and highest $(\geq 21, n=62$, highrisk group) number of T2DM risk alleles, whereas patients with 18-20 risk alleles were pooled in one group ( $n=81$, intermediate-risk group) (Fig. 1). The use of quintiles was based on a study by Lyssenko et al. [19] and allows potentially easy translation to the clinic by clear 
classification of T2DM patients. The cutoffs for the quintiles with the highest and lowest number of T2DM risk alleles fell within the group of patients with 21 and 17 risk alleles respectively. We categorized patients with 17 risk alleles to the low-risk group and 21 alleles to the high-risk group, resulting in a slightly larger number of patients in both categories than anticipated. To ascertain that our results are not solely due to study design, we also analyzed the genetic risk score as a continuous variable instead of the analysis of risk groups. Next to this genetic risk score (range 10-26), sex, age on first SU prescription, and the use of metformin were included in the multivariate analysis. Data showed similar results for both the effect size and direction for the genetic risk score (odds ratio 0.88 95\% CI: $0.76-1.02, P=0.11$ ). This suggests that with increasing number of risk alleles, the chance of achieving stable SU dose decreases.

The concept of disease-related genes influencing response to treatment is not new. For example, variation in the gene coding for the 5-hydroxytryptamine $2 \mathrm{~A}$ receptor has been associated with variation of clozapine response and increased susceptibility to schizophrenia [44,45]. Variation in the gene coding for the $\beta$-2-adrenergic receptor has been associated with airway responsiveness to $\beta$-2-receptor agonists and susceptibility to lower airway reactivity in patients with asthma $[46,47]$. Our results show that patients with a higher number of risk alleles were younger at the date of their first SU prescription. This may be the result of a more 'aggressive' form of T2DM. For many complex diseases such as T2DM, there may be multiple genetic backgrounds resulting in similar phenotypic disease, each requiring a different drug treatment. Our results support this concept, and support the use of disease-related genes in pharmacogenetic studies. We should emphasize, however, the fact that we have only begun to unravel the genetic determinants of drug response in T2DM and that although many of the genes are associated with $\beta$-cell function, the exact mechanism behind our finding remains unclear. Our results do provide some 'proof of principle' that the complex background of T2DM may ultimately result in the identification of different genetic subgroups of T2DM patients that require different pharmacotherapy. However, replication in an independent cohort and further elucidation of the causal mechanisms underlying SU response are warranted.

In conclusion, T2DM-associated risk alleles are associated with response to $\mathrm{SU}$ treatment in primary care T2DM patients. This suggests that individualization of T2DM treatment according to genetic profile may be an opportunity to improve clinical outcome.

\section{Acknowledgements}

No additional funding was received for this study. All authors declare that no conflicting interests exist.

\section{References}

1 Wild S, Roglic G, Green A, Sicree R, King H. Global prevalence of diabetes: estimates for the year 2000 and projections for 2030. Diabetes Care 2004; 27:1047-1053.

2 Krentz AJ, Bailey CJ. Oral antidiabetic agents: current role in type 2 diabetes mellitus. Drugs 2005; 65:385-411.

3 DeFronzo RA. Pharmacologic therapy for type 2 diabetes mellitus. Ann Intern Med 1999; 131:281-303.

4 Pearson ER. Pharmacogenetics and future strategies in treating hyperglycaemia in diabetes. Front Biosci 2009; 14:4348-4362.

5 Groop LC. Sulfonylureas in NIDDM. Diabetes Care 1992; 15:737-754.

6 Bouatia-Naji N, Bonnefond A, Cavalcanti-Proenca C, Sparso T, Holmkvist J, Marchand $\mathrm{M}$, et al. A variant near MTNR1B is associated with increased fasting plasma glucose levels and type 2 diabetes risk. Nat Genet 2009; 41:89-94.

7 Steinthorsdottir V, Thorleifsson G, Reynisdottir I, Benediktsson R, Jonsdottir $\mathrm{T}$, Walters GB, et al. A variant in CDKAL1 influences insulin response and risk of type 2 diabetes. Nat Genet 2007; 39:770-775.

8 Zeggini E, Weedon MN, Lindgren CM, Frayling TM, Elliott KS, Lango H, et al. Replication of genome-wide association signals in UK samples reveals risk loci for type 2 diabetes. Science 2007; 316:1336-1341.

9 Zeggini E, Scott LJ, Saxena R, Voight BF, Marchini JL, Hu T, et al. Metaanalysis of genome-wide association data and large-scale replication identifies additional susceptibility loci for type 2 diabetes. Nat Genet 2008; 40:638-645.

10 Yasuda K, Miyake K, Horikawa Y, Hara K, Osawa H, Furuta H, et al. Variants in KCNQ1 are associated with susceptibility to type 2 diabetes mellitus. Nat Genet 2008; 40:1092-1097.

11 Scott L, Mohlke KL, Bonnycastle LL, Willer CJ, Li Y, Duren WL, et al. A genome-wide association study of type 2 diabetes in Finns detects multiple susceptibility variants. Science $2007 ; 316: 1341-1345$.

12 Saxena R, Voight BF, Lyssenko V, Burtt NP, de Bakker PI, Chen $\mathrm{H}$, et al. Genome-wide association analysis identifies loci for type 2 diabetes and triglyceride levels. Science 2007; 316:1331-1336.

13 Wellcome Trust Case Control Consortium. Genome-wide association study of 14000 cases of seven common diseases and 3000 shared controls. Nature 2007; 447:661-678.

14 Sladek R, Rocheleau G, Rung J, Dina C, Shen L, Serre D, et al. A genomewide association study identifies novel risk loci for type 2 diabetes. Nature 2007; 445:881-885.

15 Winckler W, Weedon MN, Graham RR, McCarroll SA, Purcell S, Almgren P, et al. Evaluation of common variants in the six known maturity-onset diabetes of the young (MODY) genes for association with type 2 diabetes. Diabetes 2007; 56:685-693.

16 Lyssenko V, Nagorny CL, Erdos MR, Wierup N, Jonsson A, Spegel P, et al. Common variant in MTNR1B associated with increased risk of type 2 diabetes and impaired early insulin secretion. Nat Genet 2009; 41:82-88.

17 Unoki H, Takahashi A, Kawaguchi T, Hara K, Horikoshi M, Andersen G, et al. SNPs in KCNQ1 are associated with susceptibility to type 2 diabetes in East Asian and European populations. Nat Genet 2008; 40:1098-1102.

18 Cornelis MC, Qi L, Zhang C, Kraft P, Manson J, Cai T, et al. Joint effects of common genetic variants on the risk for type 2 diabetes in US men and women of European ancestry. Ann Intern Med 2009; 150:541-550.

19 Lyssenko V, Jonsson A, Almgren P, Pulizzi N, Isomaa B, Tuomi T, et al. Clinical risk factors, DNA variants, and the development of type 2 diabetes. $N$ Engl J Med 2008; 359:2220-2232.

20 Lango H, Palmer CN, Morris AD, Zeggini E, Hattersley AT, McCarthy MI, et al. Assessing the combined impact of 18 common genetic variants of modest effect sizes on type 2 diabetes risk. Diabetes 2008; 57:3129-3135.

21 Talmud PJ, Hingorani AD, Cooper JA, Marmot MG, Brunner EJ, Kumari M, et al. Utility of genetic and non-genetic risk factors in prediction of type 2 diabetes: whitehall II prospective cohort study. BMJ 2010; 340:b4838.

22 Van Hoek M, Dehghan A, Witteman JC, Van Duijn CM, Uitterlinden AG, Oostra BA, et al. Predicting type 2 diabetes based on polymorphisms from genome-wide association studies: a population-based study. Diabetes 2008; 57:3122-3128.

23 Meigs JB, Shrader P, Sullivan LM, McAteer JB, Fox CS, Dupuis J, et al. Genotype score in addition to common risk factors for prediction of type 2 diabetes. N Engl J Med 2008; 359:2208-2219.

24 Sparso T, Grarup N, Andreasen C, Albrechtsen A, Holmkvist J, Andersen G, et al. Combined analysis of 19 common validated type 2 diabetes susceptibility gene variants shows moderate discriminative value and no evidence of gene-gene interaction. Diabetologia 2009; 52:1308-1314.

25 Rutten GEHM, De Grauw WJC, Nijpels G, Goudswaard AN, Uitewaal PJM, Van Der Does FEE, et al. NHG-standard diabetes mellitus type 2. Huisarts en Wetenschap 2006; 49:137-152. 
26 Swen JJ, Wessels JA, Krabben A, Assendelft WJ, Guchelaar HJ. Effect of CYP2C9 polymorphisms on prescribed dose and time-to-stable dose of sulfonylureas in primary care patients with type 2 diabetes mellitus. Pharmacogenomics 2010; 11:1517-1523.

27 Kirchheiner J, Roots I, Goldammer M, Rosenkranz B, Brockmoller J. Effect of genetic polymorphisms in cytochrome p450 (CYP) 2C9 and CYP2C8 on the pharmacokinetics of oral antidiabetic drugs: clinical relevance. Clin Pharmacokinet 2005; 44:1209-1225.

28 Holstein A, Plaschke A, Ptak M, Egberts EH, El Din J, Brockmoller J, et al. Association between CYP2C9 slow metabolizer genotypes and severe hypoglycaemia on medication with sulphonylurea hypoglycaemic agents. Br J Clin Pharmacol 2005; 60:103-106.

29 Ragia G, Petridis I, Tavridou A, Christakidis D, Manolopoulos VG. Presence of CYP2C9*3 allele increases risk for hypoglycemia in type 2 diabetic patients treated with sulfonylureas. Pharmacogenomics 2009; 10:1781-1787.

30 Becker ML, Visser LE, Trienekens PH, Hofman A, Van Schaik RH, Stricker $\mathrm{BH}$. Cytochrome $\mathrm{P} 4502 \mathrm{C9} * 2$ and $* 3$ polymorphisms and the dose and effect of sulfonylurea in type II diabetes mellitus. Clin Pharmacol Ther 2008; 83:288-292.

31 Zhou K, Donnelly L, Burch L, Tavendale R, Doney AS, Leese G, et al. Loss-offunction CYP2C9 variants improve therapeutic response to sulfonylureas in type 2 diabetes: a Go-DARTS study. Clin Pharmacol Ther 2009; 87:52-56.

32 Pearson ER, Starkey BJ, Powell RJ, Gribble FM, Clark PM, Hattersley AT. Genetic cause of hyperglycaemia and response to treatment in diabetes. Lancet 2003; 362:1275-1281.

33 Sagen JV, Raeder H, Hathout E, Shehadeh N, Gudmundsson K, Baevre H, et al. Permanent neonatal diabetes due to mutations in KCNJ11 encoding Kir6.2: patient characteristics and initial response to sulfonylurea therapy. Diabetes 2004; 53:2713-2718.

34 Pearson ER, Flechtner I, Njolstad PR, Malecki MT, Flanagan SE, Larkin B, et al. Switching from insulin to oral sulfonylureas in patients with diabetes due to Kir6.2 mutations. N Engl J Med 2006; 355:467-477.

35 Becker ML, Aarnoudse AJ, Newton-Cheh C, Hofman A, Witteman JC, Uitterlinden AG, et al. Common variation in the NOS1AP gene is associated with reduced glucose-lowering effect and with increased mortality in users of sulfonylurea. Pharmacogenet Genomics 2008; 18:591-597.

36 Bozkurt O, De Boer A, Grobbee DE, Heerdink ER, Burger $\mathrm{H}$, Klungel $\mathrm{OH}$. Pharmacogenetics of glucose-lowering drug treatment: a systematic review. Mol Diagn Ther 2007; 11:291-302.
$37 \mathrm{Xu} \mathrm{H}$, Murray M, McLachlan AJ. Influence of genetic polymorphisms on the pharmacokinetics and pharmaco-dynamics of sulfonylurea drugs. Curr Drug Metab 2009; 10:643-658.

38 Aquilante CL. Sulfonylurea pharmacogenomics in type 2 diabetes: the influence of drug target and diabetes risk polymorphisms. Expert Rev Cardiovasc Ther 2010; 8:359-372.

39 Sesti G, Laratta E, Cardellini M, Andreozzi F, Del Guerra S, Irace C, et al. The E23K variant of KCNJ11 encoding the pancreatic beta-cell adenosine $5^{\prime}$-triphosphate-sensitive potassium channel subunit Kir6.2 is associated with an increased risk of secondary failure to sulfonylurea in patients with type 2 diabetes. J Clin Endocrinol Metab 2006; 91:2334-2339.

40 Gloyn AL, Hashim Y, Ashcroft SJ, Ashfield R, Wiltshire S, Turner RC. Association studies of variants in promoter and coding regions of beta-cell ATP-sensitive K-channel genes SUR1 and Kir6.2 with Type 2 diabetes mellitus (UKPDS 53). Diabet Med 2001; 18:206-212.

41 Holstein A, Hahn M, Stumvoll M, Kovacs P. The E23K variant of KCNJ11 and the risk for severe sulfonylurea-induced hypoglycemia in patients with type 2 diabetes. Horm Metab Res 2009; 41:387-390.

42 Pearson ER, Donnelly LA, Kimber C, Whitley A, Doney AS, McCarthy MI, et al. Variation in TCF7L2 influences therapeutic response to sulfonylureas: a GoDARTs study. Diabetes 2007; 56:2178-2182.

43 Van den Berg MJ, De Bakker DH, Spreeuwenberg P, Westert GP, Braspenning JC, Van der ZJ, et al. Labour intensity of guidelines may have a greater effect on adherence than GPs' workload. BMC Fam Pract 2009; 10:74.

44 Arranz MJ, Munro J, Birkett J, Bolonna A, Mancama D, Sodhi M, et al. Pharmacogenetic prediction of clozapine response. Lancet 2000; 355:1615-1616.

45 Inayama $\mathrm{Y}$, Yoneda $\mathrm{H}$, Sakai T, Ishida T, Nonomura $\mathrm{Y}$, Kono $\mathrm{Y}$, et al. Positive association between a DNA sequence variant in the serotonin $2 \mathrm{~A}$ receptor gene and schizophrenia. Am J Med Genet 1996; 67:103-105.

46 Holloway JW, Dunbar PR, Riley GA, Sawyer GM, Fitzharris PF, Pearce N, et al. Association of beta-2-adrenergic receptor polymorphisms with severe asthma. Clin Exp Allergy 2000; 30:1097-1103.

47 Lipworth BJ, Dempsey OJ, Aziz I. Functional antagonism with formoterol and salmeterol in asthmatic patients expressing the homozygous glycine-16 beta(2)-adrenoceptor polymorphism. Chest 2000; 118:321-328. 